\title{
Design and development of the apply force tester based on pneumatic servo control
}

\author{
Xu Xiaoguang ${ }^{1, a}$, Li Xu ${ }^{1}$, Liu Binbin ${ }^{1}$, Zhang Bin ${ }^{1}$ and Yin Lijuan ${ }^{1}$ \\ ${ }^{1}$ Shenzhen Entry-Exit Inspection and Quarantine Bureau, China
}

\begin{abstract}
Based on the requirement of compression and tension test in toy safety standard, combined with the PLC technology, taken pneumatic servo system to design and develop the automatic apply force tester and realized to precise control of the force and displacement in test process. This paper is emphasized on mechanism design, pneumatic servo system structure, software control principle and function characteristics of the force tester. It pointed out use the Pneumatic Servo can enhance the precision and the level of whole system's autoimmunization in testing.
\end{abstract}

\section{Introduction}

In order to ensure the health and safety of the children, the toy import and export must be tested by a series of relevant standards, and the testing of the tension and impression is an important part in the mechanical and physical properties of the toys. National safety of toys GB6675, international standard ISO8124, the European standard EN71-1, American Standard ASTM F963-11, the toys for impression, tension test have the corresponding requirements. Such as the European standard EN71-1 in the impression testing requirements for: Place the toy on a horizontal rigid surface with the part of the toy to be tested uppermost. Apply a compression force of $(110 \pm 5) \mathrm{N}$ through a rigid metal disc with a diameter of $(30 \pm 1.5) \mathrm{mm}$ to the area to be tested. Apply the force gradually over a period of approximately $5 \mathrm{~s}$ and Maintain the force for $10 \mathrm{~s}$. Tension test requirements for: Apply a force of $(90 \pm 2) \mathrm{N}$ when the largest accessible dimension of the component to be gripped and tested is greater than $6 \mathrm{~mm}$, the tension required to be uniformly applied within the $5 \mathrm{~S}$, and parallel to the test parts of the spindle, and then maintain $10 \mathrm{~s}\left[{ }^{1][2]}\right.$.

At the present stage, the test is carried out by manual pulling force. In the process of the application of force, it will bring great deviation because of applying force is not precision. At the same time, because of the manual way of the test is very inconvenient, It can bring man-made damage to the toy in the test process, seriously affect the test results.

With the development of industrial automation technology, electrical-pneumatic proportional and servo control system has been widely used. Application of electrical-pneumatic servo positioning control system can realize the multi-point stepless positioning and stepless speed regulation, and can realize the continuous

\footnotetext{
${ }^{\mathrm{a}}$ Xu Xiaoguang: xu.xg@163.com
}

adjustable speed of the cylinder. The apply force tester adopts pneumatic servo valve system, which can meet the requirements of tension, impression testing in toy safety standards: EN71-1, GB6675, ISO8124, and ASTM F963.

\section{Structure design}

The automatic apply force tester adopts the closed-loop control system, the tester is composed of the mechanical loading part, the electrical control part, the gas source control part, the data processing and the display part. The function of each part is as follows: the mechanical loading part mainly is the support to be tested toys, according to the procedure request to carry on the test work; the electrical control part is mainly composed of the PLC and the position sensor, completes the action logic control. Gas source control part is mainly composed of the pneumatic servo valve and the precision pressure regulator valve, mainly accurate controls the gas supply pressure. Data processing and display part realizes the main data processing, comparison, output and display functions. Schematic diagram of the test apparatus is shown in Figure 1.

The whole mechanical system consists of test machine platform 1 , linear guide rail 2 , damping cylinder 3 , force cylinder 4 , pressure sensor 5 , position sensor 6 , toy assembly clamp 7 , apply force connecting rod 8 , touch screen 9 and so on. The main body of the tester is to install the test work and the implementation part. Linear guide rail 2 is arranged in the cylinder 4 , mainly to meet some toy testing after the force applying cylinder 4, deformed, cushion cylinder 4 shifted in position and setting. The role of the damping of the cylinder 3 is to increase the damping of the cylinder 4 in the process of sliding, so that the change process is slow. The application of cylinder 4 is mainly to realize the pressure and tension of the tested toys. Pressure sensor 5 is a real- 
time monitoring of the force which loads on the toy. The position sensor 6 is mainly to detect the displacement of the toys in the process of the deformation or damage. The clamp of the toy assembly is used for the fixed test toy, which is convenient for testing. Touch screen 8 can display the value of the pressure force in the test process in real time, and the value of the displacement. It can also input the test value which the test standard requires. Force application rod connecting rod has a conversion chuck, when carrying out the impression test, connected to a rigid circular metal pressure head. When carry out the tension test, it connected to a metal pull hook. Force connecting rod is connected with the cylinder 4, movement with the cylinder when carry out the tension or impression test.
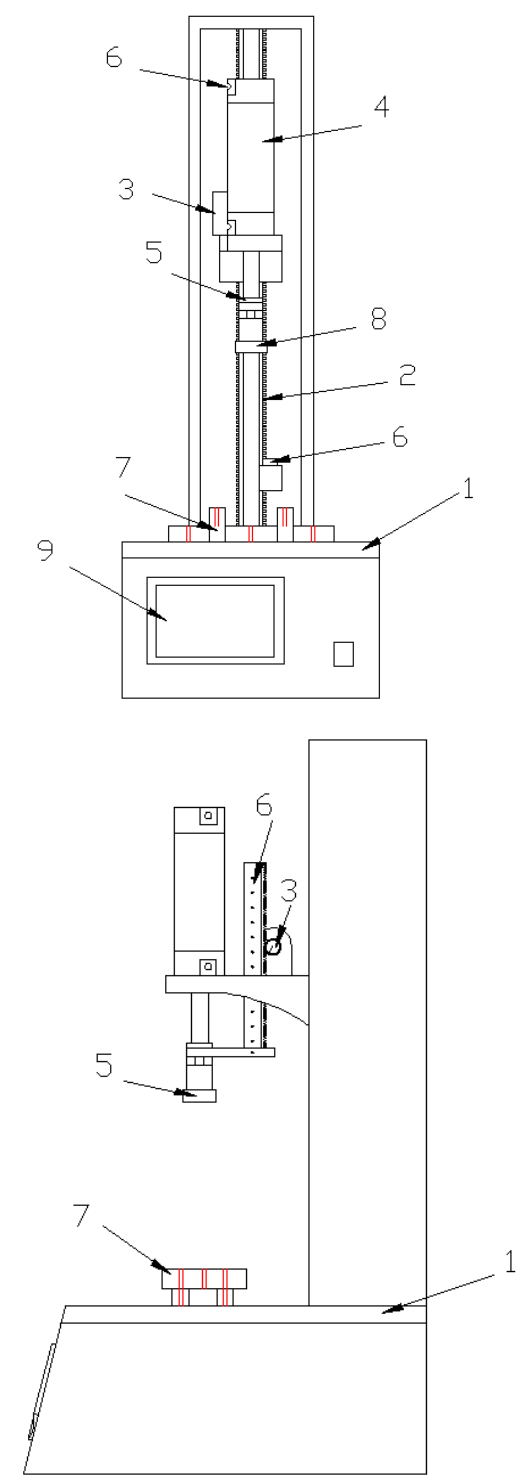

Figure 1. Mechanical structure of tester

$\begin{array}{lll}\text { 1. Machine platform } & \text { 2. Linear guide rail } 3 \text {. Damping cylinder }\end{array}$ 4. Force cylinder 5. Pressure sensor 6.Position sensor 7.Toy assembly clamp 8.Apply force rod 9.Touch screen

\section{Sever control system of tester}

According to the toy standard, the force tester has the following functions: 1) The continuous pressure testing of the surface of the toy; 2) the tensile test of the part of the toy; 3) continue to apply force and maintain for a while. At the same time, when the toy damages, issue the sound and light alarm.

According to the requirements and testing method, the system is composed of the pneumatic servo valve controlled by pneumatic, load sensor, fault alarm sensor and controller. In the standard, the maximum force of the compression is $110 \mathrm{~N}$ and the maximum tension force is $90 \mathrm{~N}$. The error range was $5 \%$. According to the size of the force, the use of TH1 type pressure sensor, capacity is $100 \mathrm{~kg}$, the nonlinear error of the $0.3 \% \mathrm{FS}$, the hysteresis error $<0.3 \% \mathrm{FS}$, the repeatability error $<0.3 \% \mathrm{FS}$, the precision of the sensor is completely in line with the standard requirements. According to the requirements, the control of the closed-loop force control, in the case of the use of the cylinder, the use of pneumatic servo valve. The test gauge pad cylinder is a cylinder with a diameter of $63 \mathrm{~mm}$, calculated by the pressure $0.5 \mathrm{MPa}$, the use of the United States MAC pneumatic servo valve, the valve model is PPC5C-BBA-IB, the highest pressure $0.7 \mathrm{MPa}$, the highest control accuracy can be controlled at $\pm 1 \%$, the input signal is $4-20 \mathrm{~mA}^{[3]}$.

In the control system of the system, the input signal is: the sensor signal and the force sensor signal (4-20mA). The output signal is: servo drive signal (analog 4-20mA), cylinder solenoid valve signal. According to the requirements can display real-time state and set parameters. According to the contents of the control, select the PLC of Panasonic FP0, the amount of the switch according to the number of control C14RS, A21, the module has two analog input (A/D), one analog output(D/A), At the same time, the output signal is AFP0421, D/A, 0-20mA. According to the display settings, select the FP0 to match the touch screen.

Control system connection diagram shown in Figure 2.

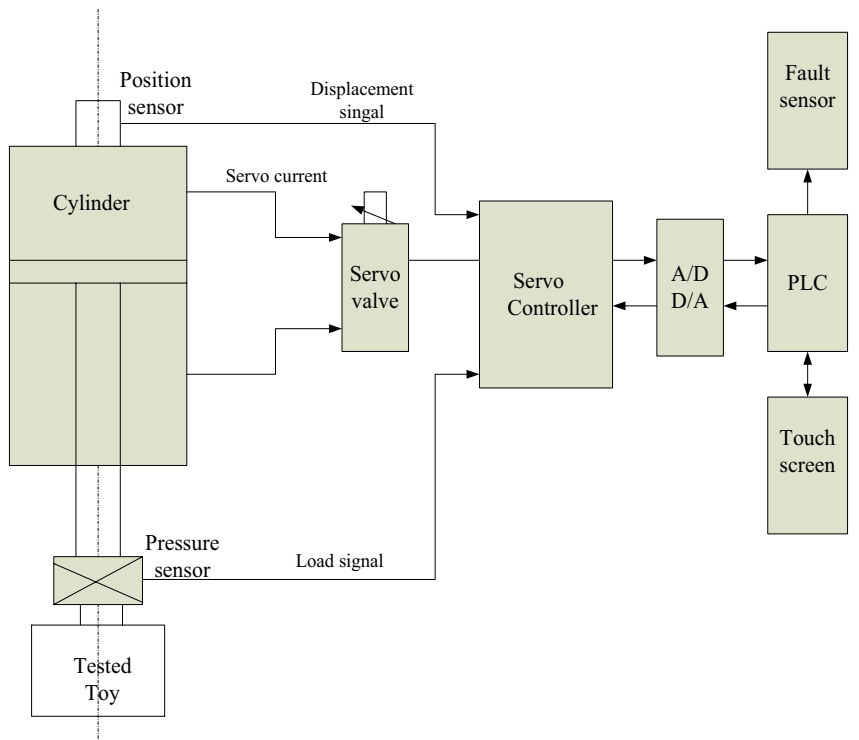

Figure 2. Servo control system 


\section{System control principle}

\subsection{Basic structure of servo control system}

Servo control valve with PLC or microcomputer control technology can meet all kinds of control requirements. The servo control system is basically formed as shown in Figure 3. The actuator element in the diagram can be an element that can be converted into mechanical energy by the pressure of the air, such as a cylinder, a gas motor, a container, and a nozzle. Servo control valve as the interface element of the system's electrical and pneumatic conversion, supplies to control air pressure energy of the actuator. As the interface of man-machine, the controller plays a role in controlling the quantity of the servo control valve. It can be single chip microcomputer or PLC controller. Servo control valve has high accuracy, general $0.5-2.5 \% \mathrm{FS}$. Even if the negative feedback system is not composed of various sensors, it can get very ideal control effect, but it can not restrain the influence of the parameters of the controlled object and the external interference. In order to control the accuracy of the application, the use of various sensors to form a negative feedback to further improves the system's control accuracy ${ }^{[4]}$.

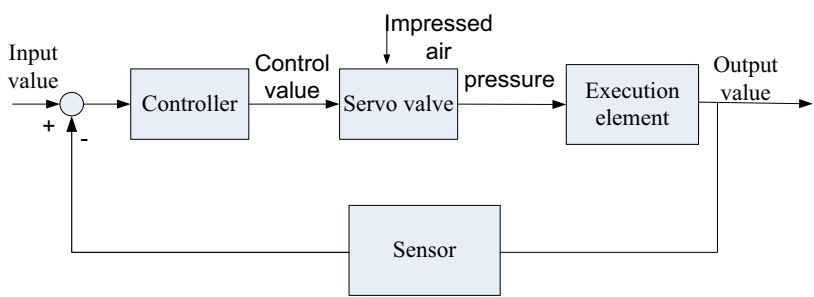

Figure 3. Basic components of servo control system

\subsection{Servo control principle}

In the control system, the microcomputer or PLC is used as the controller, and the $\mathrm{A} / \mathrm{D}$ converter can be directly driven, and the servo control system can be composed by using standard cylinder and position sensor. In Figure 3, the target value is controlled by the controller to the servo valve, and the motion control of the cylinder is controlled by the controller. The displacement of the cylinder is detected by the position sensor and fed back to the controller. The controller is based on the displacement feedback of the cylinder, and the velocity and acceleration feedback are calculated. Also according to the operating conditions (load mass, bore, stroke and valve size), automatically calculate the optimal signal control value, and the role of the servo control valve to achieve closed-loop control. The controller is connected with the programmable controller to realize the sequence of other systems ${ }^{[5]}$.

\subsection{Force control principle}

D/A PLC conversion module output analog controls output pressure of servo valve, changing the output pressure will change the cylinder pressure. The cylinder produce the force $\mathrm{F}=\mathrm{P} * \mathrm{~S}, \mathrm{P}$ is the pressure, $\mathrm{S}$ is the area of action, $\mathrm{P}$ is working pressure ${ }^{[6]}$.

The formula for calculating the output of the cylinder theory is:

$$
F=\frac{\pi}{4} \times D^{2} \times P
$$

$\mathrm{F}=$ Output Force

$\mathrm{D}=$ Diameter of Cylinder

$\mathrm{P}=$ Air Pressure

In test, $\mathrm{F}(\mathrm{N})$ is:

Impression : $F(I)=\frac{\pi}{4} \times D^{2} \times P$

Tension: $\quad F(T)=\frac{\pi}{4} \times\left(D^{2}-d^{2}\right) \times P$

D: Diameter of Cylinder ( $\mathrm{mm}$ )

$\mathrm{d}$ : Diameter of Piston rod $(\mathrm{mm})$

P: Air Pressure (MPa)

\section{Software design}

The testing process is divided into 4 steps: first, to increase the pressure in the actuator chamber, test the process parameters curve, and then reduce the pressure in the actuator cavity, and then test the return parameter curve, control process, as shown in Figure 4. Operator installed the tested toys, start test button, PLC according to the user setting, by D/A module output $4-20 \mathrm{~mA}$ analog signal, through the gas dynamic servo valve control the execution chamber pressure, stable pressure, PLC through the ad module read the signal of displacement sensor and a pressure sensor; again by gas dynamic servo valve according to set the parameters of gradually increasing chamber pressure to perform, and record the signal of displacement sensor and pressure sensor until it reaches the maximum set pressure value. Then, the pressure in the chamber is reduced, and the signal of the displacement and pressure sensor is recorded until the minimum pressure is reached. Then, the PLC will test the data through the interface to display the touch screen. In the end, the product is qualified by setting the standard parameters ${ }^{[7][8]}$. 


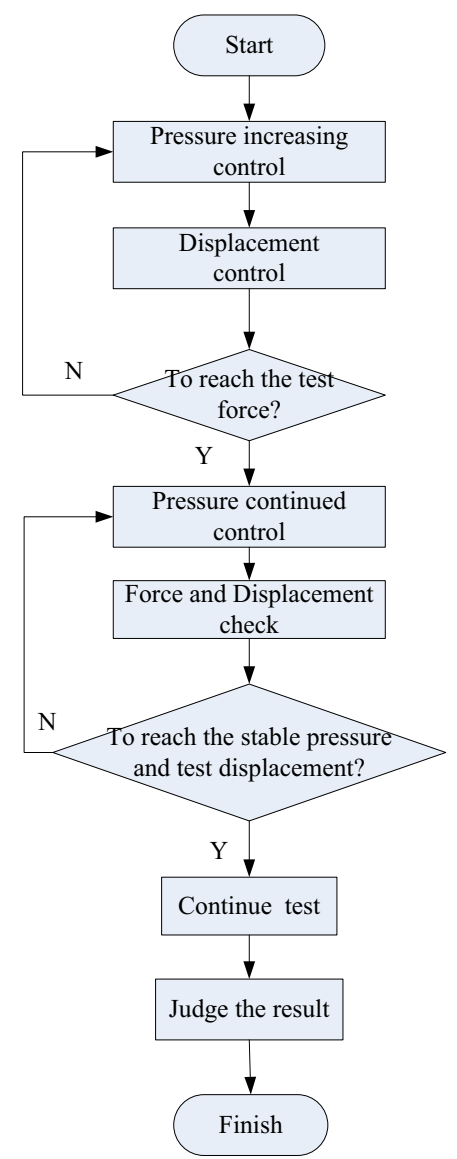

Figure 4. Software system of the tester

\section{Functional properties}

(1) One tester has multiple functions: the test instrument to meet the requirements of a number of standard apply force test, to achieve the impression and tension test on a single instrument. Mainly meet the standards: EN711:2014, the national toy safety standards GB6675.2-2014, international toy safety standards ISO8124-1:2014, American toy standards F963-11 ASTM, etc..

(2) Fixed and adjusted of the toys easily: on the platform of the measuring instrument can be in accordance with a variety of test fixture, to meet the requirements of different shapes and types of toys.

(3) The test process automation: because the testing process is measured, the load mechanism, the PLC, the pneumatic servo system, the sensor and the human machine hand touching screen constitute a closed loop testing process, the automatic control of the testing process is realized based on the accurate speed.

(4) High accuracy, fast speed, strong stability. Because of the fast speed, high precision and high accuracy of PLC processing, the fast response of servo control technology makes the testing process fast and accurate.

\section{Conclusions}

The automatic apply force tester adopts PLC and pneumatic servo system, which ensures the stability of the test system, the fast response and high precision.
Using this tester can not only reduce the labor intensity, but also greatly improve the detection efficiency, improve the accuracy of detection results, improve the automation level of inspection work. The instrument has wide application prospect in the safety testing of toys.

\section{References}

1. BS EN 71-1:2014, Safety of toys -part 1: Mechanical and Physical properties.

2. BS ISO 8124-1:2014 Safety of toys -part 1: Safety aspects related to mechanical and physical properties.

3. J.Jihai, Hydraulic and pneumatic transmission. (2002).

4. Q.Rongqing, Servo system. (2006).

5. S.Shibing, Communication between Mitsubishi PLC and PC, J. Control \& Automation, 4(2006).

6. Y.Hanqi, Electric control and PLC application technology. (2003).

7. Y.Qingquang, PLC principle and system design, Beijing. (2004).

8. Z.Yuehua, PLC technology and application (1998). 